\title{
ACHADOS TOMOGRÁFICOS EM 1000 PACIENTES CONSECUTIVOS COM ANTECEDENTES DE CRISES EPILÉPTICAS
}

\author{
Ana Paula Trentin', Hélio A.G. Teive², Mario H. Tsubouchi', \\ Luciano de Paola4, Guilberto Minguetti ${ }^{5}$
}

\begin{abstract}
RESUMO - Analizamos retrospectivamente as tomografias computadorizadas (TC) de crânio de 1000 pacientes consecutivos, com história clínica de crises epilépticas classificadas do ponto de vista clínico em generalizadas (CG) e parciais (CP). Nossos resultados mostraram a presença de CG em 70,7\% dos pacientes, sendo $57,1 \%$ do sexo masculino e $42,9 \%$ do sexo feminino e, CP em $29,3 \%$, sendo $60,0 \%$ do sexo maculino e $40,0 \%$ do sexo femninino. As faixas etárias de maior incidência foram entre 0 a $10(31,0 \%)$ e 11 a $20(21,8 \%)$ anos nos pacientes com CG e 0 a $10(24,5 \%), 21$ a $30(16,7 \%)$ e 31 a 40 anos $(18,4 \%)$ nos pacientes com CP. Os resultados tomográficos no grupo com CG foram: normais (48,8\%) e alterados em $51,2 \%$, sendo esses achados definidos como calcificações/cisticercose $(14,0 \%)$, neurocisticercose/cistos $(9,6 \%)$ hidrocefalia $(4,4 \%)$, infarto $(4,2 \%)$, indefinido $(4,0 \%)$, tumor $(2,5 \%)$ entre outros $(12,5, \%)$. Nos pacientes com CP os exames tomográficos foram normais em $37,4 \%$, alterados em $62,7 \%$, tendo os diagnósticos de neurocisticercose/cistos $(12,2 \%)$, calcificações/cisticercose (11,2\%), tumor (10,5\%), indefinido (8,1\%), infarto $(5,4 \%)$, hidrocefalia $(3,7 \%)$ e outros $(11,6 \%)$. Salientamos a importância da TC em pacientes com epilepsia, particularmente para o diagnóstico de neurocisticercose.
\end{abstract}

PALAVRAS-CHAVE: epilepsia, tomografia computadorizada, neurocisticercose.

\section{CT findings in 1000 consecutive patients with seizures}

ABSTRACT - We analized the cranial computorized tomography scans (CT) of 1000 consecutive patients with the history of seizures. The seizures were classified in generalized tonic-clonic seizures (GS) and partial seizures (PS) on the basis of the clinical semiology, as it was described by the patients and family members. Seizure types were correlated with age group, sex and CT findings. GS were observed in $70.7 \%$ of the patients and PS in $29.3 \%$. An increased incidence of GS was noted among the age groups 0 to $10 \mathrm{yrs}(31 \%)$ and 11 to $20 \mathrm{yrs}$ (21.8\%). For PS the 0 to 10 yrs (24.5\%), 21 to 30 yrs (16.7\%) and 31 tp 40 yrs (18.5\%) age groups were the most prevalent groups. The CT scan findings for the GS group were the following: normal studies $48.8 \%$ of the patients, calcifications/cysticercosis (14.0\%), neurocysticercosis/cysts $(9.6 \%)$, hydrocephalus (4.3\%), ischemia $(4.2 \%)$, non-definite lesions (4.1\%), tumor (2.5\%), and others (12.5\%). In the PS group we found $37.4 \%$ of normal studies, calcifications/cysticercosis (11.2\%), neurocysticercosis/cysts (12.2\%), tumor (10.5\%), ischemia (5.4\%), hydrocephalus (3.7\%), non-definite lesions (8.1\%) and others (11.6\%). We emphasize the importance of CT scan imaging in patients presenting with seizures, particularly in the diagnosis of cysticercosis.

KEY WORDS: epilepsy, computed tomography, cysticercosis.

A determinação da etiologia das crises epilépticas mudou significativamente a partir da introdução dos métodos de neuroimagem, notadamente a tomografia computadorizada (TC) cerebral. Na década de 70, Gastaut ${ }^{1}$ já propunha uma avaliação anátomo-eletroclínica para a classificação das síndromes epilépticas, tamanha a relevância da TC para o diag- nóstico etiológico das mesmas. Atualmente acredita-se que o melhor método para investigação de alterações estruturais em epilepsia seja a ressonância magnética (RM) $)^{2,3}$. A TC, contudo, ainda se apresenta como um importante instrumento diagnóstico, particularmente em função da alta sensibilidade na demonstração das calcificações intraparenquimato-

Estudo realizado no CETAC - Centro de Tomografia Computadorizada e Ressonância Magnética, Curitiba PR, Brasil: ${ }^{1}$ Médico Residente em Neurologia da Universidade Federal do Paraná (UFPR); ${ }^{2}$ Professor Assistente de Neurologia da UFPR, Médico do CETAC, ${ }^{3}$ Neurologista, Médico do CETAC, ${ }^{4}$ Neurologista, Médico do CETAC, Diretor do Programa de Cirurgia de Epilepsia do Hospital das Clinicas-UFPR; ${ }^{5}$ Professor Adjunto de Neurologia da UFPR, Diretor Médico do CETAC.

Recebido 8 Julho 2001, recebido na forma final 29 Novembro 2001. Aceito 7 Dezembro 2001. 
sas associadas à neurocisticercose (NC), doença de grande incidência nos paises em desenvolvimento e que se manifesta principalmente na forma de crises epilépticas ${ }^{4-7}$.

Neste estudo, demonstramos os achados tomográficos em 1000 pacientes consecutivos com história de crises epilépticas, enfatizando o potencial deste método diagnóstico na definição etiológica das crises.

\section{MÉTODO}

Foram analisadas retrospectivamente as TC realizadas em 1000 pacientes consecutivos com história clínica de crises epilépticas, realizadas no Centro de Tomografia Computadorizada e Ressonância Magnética (CETAC), Curitiba, Paraná, no período de 1978 a 1990.

Foram incluídos todos os pacientes com história clínica compatível com crises epilépticas, e encaminhados para exame tomográfico por este motivo. As crises foram definidas como crises parciais (CP), subdivididas em parciais simples e complexas, e crises generalizadas tônico-clônicas (CG) com base exclusiva nos critérios semiológicos da Classificação Internacional das Crises Epilépticas ${ }^{8,9}$.

Todos os exames foram interpretados por radiologistas e neuroimagenologistas, com o resgistro de achados tomográficos e sua correlação com idade, sexo e tipo de crise.

Para os fins deste levantamento, o diagnóstico tomográfico de neurocisticercose (NC) foi definido pela presença de lesões intra-parenquimatosas encefálicas císticas, com ou sem captação do contraste iodado, de forma anular, com ou sem edema perifocal e associadas ou não a presença de calcificações intraparenquimatosas. Foram incluídas também as formas de NC racemosa (sub-aracnóidea e ventricular). As calcificações intraparenquimatosas puntiformes, isoladas ou múltiplas foram consideradas sugestivas de NC. Outras calcificações intraparenquimatosas encefálicas (isoladas ou múltiplas), não sugestivas de NC, foram categorizadas como "Indefinidas" ou "Outros", quando francamente sugestivas de outras patologias que não NC, tais como infecções do sistema nervoso central por toxoplamose, rubéola, citomegalovírus, além de esclerose tuberosa.

\section{RESULTADOS}

Entre os 1000 pacientes da amostragem, 707 (70,7\%) apresentaram história sugestiva de crises CG e 293 (29,3\%) episódios sugestivos de CP.

Não foi observada diferença significativa entre a faixa etária dos pacientes com CG (404 homens e 303 mulheres) e CP (176 homens e 117 mulheres) (Tabela 1).

Os exames de TC foram normais em 345 pacientes $(48,8 \%)$ com CG e 109 pacientes $(37,3 \%)$ com $\mathrm{CP}$, sendo assim a normalidade o principal achado tomográfico independente do tipo de crise observada na história clínica. No grupo de pacientes com CG Havia 362 pacientes ( $51,2 \%$ dos casos) com exa-
Tabela 1. Distribuição dos pacientes estudados que apresentavam CG e CP conforme a idade.

\begin{tabular}{lcccc}
\hline & \multicolumn{2}{c}{$\mathrm{CG}$} & \multicolumn{2}{c}{$\mathrm{CP}$} \\
Idade & $\mathrm{n}^{\circ}$ & $(\%)$ & $\mathrm{n}^{\circ}$ & $(\%)$ \\
\hline $0-10$ & 219 & 31,0 & 72 & 24,5 \\
$11-20$ & 154 & 21,8 & 47 & 16,0 \\
$21-30$ & 109 & 15,4 & 49 & 16,7 \\
$31-40$ & 88 & 12,5 & 54 & 18,5 \\
$41-50$ & 55 & 7,8 & 27 & 9,2 \\
$51-60$ & 43 & 6,0 & 24 & 8,2 \\
$61-70$ & 29 & 4,1 & 12 & 4,1 \\
$71-80$ & 5 & 0,7 & 8 & 2,8 \\
$81-90$ & 5 & 0,7 & 0 & 0,0 \\
\hline
\end{tabular}

Tabela 2. Distribuição dos pacientes estudados conforme a etiologia das CG e CP no laudo das TC.

\begin{tabular}{lcccc}
\hline & \multicolumn{2}{c}{ CG } & \multicolumn{2}{c}{ CP } \\
Laudo TC & $\mathrm{n}^{\circ}$ & $(\%)$ & $\mathrm{n}^{\circ}$ & $(\%)$ \\
\hline Normal & 345 & 48,8 & 109 & 37,3 \\
Calcificações/NC & 99 & 14,0 & 33 & 11,2 \\
Neurocisticercose/cistos & 68 & 9,6 & 36 & 12,2 \\
Hidrocefalia & 31 & 4,3 & 11 & 3,7 \\
Tumor & 18 & 2,5 & 31 & 10,5 \\
Infarto & 30 & 4,2 & 16 & 5,4 \\
Indefinido & 29 & 4,1 & 24 & 8,1 \\
Outros & 87 & 12,5 & 33 & 11.6 \\
\hline
\end{tabular}

Tabela 3. Distribuição dos pacientes que apresentavam CG e CP conforme localização da lesão encefálica na TC.

\begin{tabular}{lcccc}
\hline $\begin{array}{l}\text { Localização da } \\
\text { lesão na TC }\end{array}$ & \multicolumn{2}{c}{ CG } & \multicolumn{3}{c}{ CP } \\
\hline Múltiplas/difusas & 183 & 49,5 & 50 & 28,8 \\
Temporal direita & 25 & 6,7 & 22 & 12,7 \\
Temporal esquerda & 23 & 6,2 & 19 & 10,9 \\
Frontal direita & 18 & 4,9 & 13 & 7,5 \\
Parietal direita & 9 & 2,4 & 7 & 4,0 \\
Parietal esquerda & 16 & 4,3 & 14 & 8,0 \\
Outros & 96 & 26.0 & 49 & 28,1 \\
\hline
\end{tabular}


mes tomográficos de crânio anormais, enquanto que no grupo de pacientes com CP este número foi de 184 pacientes ( $62,7 \%$ dos casos).

Entre os exames anormais, as alterações compatíveis com o diagnóstico de NC (formas cística, com ou sem sinais de degenração ou calcificada) corresponderam a maior parte dos achados $(23,5 \% \mathrm{em}$ média, sendo $23,6 \%$ no grupo com CG e $23,4 \%$ no grupo com CP) (Tabela 2). A localização anatômica das lesões encefálicas encontra-se discriminada na Tabela 3.

\section{DISCUSSÃO}

Nossos resultados demonstraram maior frequência de crises epilépticas, tanto generalizadas como parciais, nos pacientes do sexo masculino, maior incidência de crises epilépticas generalizadas nos pacientes mais jovens e maior frequência de achados tomográficos sugestivos de NC em ambos os grupos dos pacientes estudados, evidenciando distribuição difusa das lesões detectadas na TC, tanto nos pacientes com CG como nos com CP. O estudo demonstrou que a maioria dos pacientes era do sexo masculino, apesar da literatura relatar maior acometimento do sexo feminino na NC e de esta ser a principal causa de crises epilépticas ${ }^{10,11}$.

A maioria dos pacientes $(52,8 \%)$ acometidos por CG pertencia a faixa etária mais baixa, entre 0 a 20 anos. Este é o principal tipo de crise quando a causa não é uma lesão cerebral visualizada à TC e sim a uma predisposição constitucional (genética ou idiopática) à crise epiléptica ou talvez a um distúrbio de migração não detectado nas técnicas convencionais de imagem ${ }^{12}$, bem como história prévia de paralisia cerebral ou meningite. Esse dado torna-se mais evidente quando analisamos que $48,8 \%$ dos pacientes classificados clinicamente como portadores de CG, apresentavam TC normal, diferentemente dos que apresentavam $\mathrm{CP}$, onde a normalidade como laudo da TC correspondia a 37,4\%. Gastaut, em 1976, demonstrou que $90 \%$ dos pacientes analisados em um estudo retrospectivo apresentavam TC normal quando portadores de CG primárias e esse número caia para $37 \%$ nos pacientes com $\mathrm{CP}^{1}$. Contudo, relatase uma variação importante, de 30 a $60 \%$, na sensibilidade da TC na detecção de lesões estruturais nos pacientes que apresentam crises epilépticas ${ }^{1,13}$. Há que se discutir, contudo, as dificuldades na correta classificação das crises epilépticas, tanto na sua diferenciação entre CP e CG (ou ainda as secundárias generalizadas), como no diagnóstico diferencial com outras causas de perda súbita e transitória da cons- ciência, como as síncopes e os eventos não-epilépticos. Neste tipo de estudo estes problemas podem dificultar as interpretações gerais dos resultados. Contudo, acreditamos que o número expressivo de pacientes com cistos e calcificações avaliados em TC pode dar uma idéia global das causas de crises epilépticas em nosso meio, particularmente o diagnóstico de NC.

Houve, nas últimas décadas, com a criação dos métodos de imagem, uma verdadeira revolução no diagnóstico da epilepsia ${ }^{13}$. A utilização da TC, ajudou a elucidar a relação comum entre NC e crises epilépticas, tanto auxiliando o diagnóstico como orientando sobre a melhor conduta a ser tomada ${ }^{14}$. A NC é uma doença de grande prevalência em nosso país, representando a mais freqüente parasitose do sistema nervoso central, devido a nossa condição de país em desenvolvimento ${ }^{10,15-17}$. Localidades em nosso país, antes sem relato da doença, constataram ser a NC o diagnóstico de mais de $1 \%$ de todos pacientes internados após a introdução da tomografia computadorizada em serviço hospitalar ${ }^{16}$. Arruda demonstrou, após a avaliação de 210 TC de pacientes epilépticos, ser a NC a causa mais comum identificável de epilepsia em nosso meio ${ }^{4}$. Nossos dados demonstraram resultado semelhante. Observamos como achado tomográfico a presença de lesões típicas de NC (seja na forma ativa : cistos intraparenquiamotosos, ou na forma inativa: calcificações) em $23.6 \%$ e $23.4 \%$ nos pacientes com CG e $\mathrm{CP}$, respectivamente. Talvez esta informação explique a incidência de lesões registradas nas TC dos pacientes com crises epilépticas, uma vez que este é um método sensível para detectar lesões de NC, principalmente no referente às calcificações intra-parenquimatosas ${ }^{18-20}$.

Os achados tomográficos característicos de NC permitem classificar a doença em formas ativa e inativa, dependendo do estágio de evolução das lesões ${ }^{21}$. Machado e colaboradores, propuseram uma nova classificação tomográfica procurando estabelecer vínculo entre as imagens observadas à TC e a evolução biológica dos cisticercos ${ }^{22}$. Nosso estudo não caracterizou os diversos estágios da evolução dos cistos, como a presença de edema e captação anular pelo contraste na fase de degeneração, visto não ser nosso objetivo a correlação da atividade da doença com os demais dados clínicos.

Outras causas de crises epilépticas encontradas, com menor freqUência, tanto no grupo com crises generalizadas como parciais foram: hidrocefalia, infarto e tumores cerebrais. Deve-se ressaltar que no 
grupo de pacientes com crises epilépticas parciais o diagnóstico de tumor cerebral foi encontrado em $10,5 \%$ dos casos, representando a segunda causa mais comum de crises epilépticas neste estudo.

Quanto a frequência da localização das lesões, observamos um predomínio de lesões múltiplas/difusas no laudo tomográfico, chegando a quase $50 \%$ nos pacientes acometidos por CG e $28.8 \%$ nos que apresentavam CP. A literatura relata a presença de lesões únicas em mais de $50 \%$ dos pacientes com NC, dado diferente dos nossos resultados, porém possivelmente explicado pela variedade de outras causas encontradas nos laudos tomográficos e pela invasão difusa no parênquima cerebral por múltiplos cisticercos ${ }^{14}$.

Os resultados do presente estudo servem para reforçar a importância da TC no diagnóstico de NC, já que esta entidade representa em nosso meio a causa mais freqüente de crises epilépticas secundárias.

\section{REFERÊNCIAS}

1. Gastaut H, Gastaut JL. Computerized transverse axial tomography in epilepsy. Epilepsia 1976;17:325-336.

2. Jackson GD. New techniques in magnetic resonance and epilepsy. Epilepsia 1994; 35(Suppl 6):S2-S13.

3. Spencer SS. The relative contributions of MRI, SPECT, and PET imaging in epilepsy. Epilepsia 1994;35(Suppl 6):S72-S89.

4. Arruda WO. Etiology of epilepsy: a prospective study of 210 cases. Arq Neuropsiquiatr 1991;49:251-254.

5. Albuquerque ES, Galhardo I. Neurocisticercose no Estado do Rio Grande do Norte: relato de oito casos. Arq Neuropsiquiatr 1995;53: 464-470.
6. Simas ZMG, Kameoka RFA. Neurocisticercose. J Bras Med 1992;63:15-37.

7. Takayanagui OM, Jardim E. Aspectos clínicos da neurocisticercose. Arq Neuropsiquiatr 1983;41:50-63.

8. Dreyfuss D. Proposal for revised clinical and eletroencephalografic classification of epileptic seizure. Epilepsia 1981;22:489.

9. Commission on Classification and Terminology of the International League against Epilepsy. Proposal for revised clinical and electroencephalographic classification of epileptic seizures. Epilepsia 1981;22: 489501.

10. Narata AP, Arruda WO, Uemura E, et al. Neurocisticercose: diagnóstico tomográfico em pacientes neurológicos. Arq Neuropsiquiatr 1998; 56:245-249.

11. Takayanagui OM. Neurocisticercose: evolução clínico-laboratorial de 151 casos. Arq Neuropsiquiatr 1990;48:1-10.

12. Savic I, Seitz RJ, Pauli S. Brain distortions in patients with primarily generalized tonic-clonic seizures. Epilepsia 1998;39: 364-370.

13. Kuzniecky RI. Neuroimaging in pediatric epilepsy. Epilepsia 1996;37(Suppl 1):S10-S21

14. Commission on Tropical Disease of the International League Against Epilepsy. Relationship between epilepsy and tropical disease. Epilepsia 1994;35: 89-93.

15. Arruda WO, Camargo NJ, Coelho RC. Neurocysticercosis: an epidemiological survey in two small rural communities. Arq Neuropsiquiatr 1990;48:419-424.

16. Coêlho-Gonçalves TD, Coêlho MDG. Cerebral cysticercosis in Campina Grande, Paraíba-northern Brazil. Arq Neuropsiquiatr 1996;54:94-97.

17. Teive HAG, Minguetti G, Sasaki MGM, Lopes CE, Carvalho MTM, Szpeiter N. Neurocisticercose. artigo de revisão. Rev Bras Neurol 1997; 33:147-153.

18. Arruda WO, Meneses MS, Antoniuk AS. Neurocisticercose: diagnóstico e tratamento. Rev bras Neurol 1996;32:15-20.

19. Carpio A, Escobar A, Hauser WA. Cysticercosis and epilepsy: a critical review. Epilepsia 1998:39: 1025-1040

20. ILAE Commission Report. Guidelines for neuroimaging evaluation of patients with uncontrolled epilepsy considered for surgery. Epilepsia 1998;39:1375-1376.

21. Del Brutto OH, Otelo J, Román GC. Neurocysticercosis: a clinical handbook. Lisse: Swetts Zeitlinger, 1998.

22. Machado LR, Nobrega JPS, Barros NG, Livramento JA, Bacheschi LA, Spina-França A. Computed tomography in neurocysticercosis: a 10year long evolution analysis of 100 patients with an appraisal of a new classification. Arq Neuropsiquiatr 1990;48:414-418. 